\title{
Neuroradiologic Applications in the Assessment of Alzheimer's Disease and the Potential Implications of Brain Imaging in Forensic Psychiatry
}

\author{
Ayden Jacob ${ }^{1}$ \\ 1. Interventional Radiology and Oncology, UCSF School of Medicine
}

$\square$ Corresponding author: Ayden Jacob, brainanalytics1@gmail.com

Disclosures can be found in Additional Information at the end of the article

\section{Abstract}

The field of radiology has undergone a dramatic change in the last decade due to the innovations manifesting from domains, such as electrical engineering, biomedical engineering, and computer sciences. The capability of a radiologist to gain deep insight into the working mechanisms of the brain at the anatomical and physiological level is unprecedented. Molecular and biochemical pathways undetected hitherto are now imaged consistently to provide a refined diagnosis and more reliable therapeutic plan. Although the milestones in radiology influence and propel the progress of a variety of medical domains, neuropsychiatry benefits from these technological improvements in a tangible, concrete, and translational manner. The manner and reasons, by which a brain may begin to develop abnormal functions, remains elusive. However, biomedical imaging has provided clinicians and scientists alike the ability to gain a deeper insight into fundamental substrates of the workings of the brain. Consequently, the biomedical community addressing the mental health community is now better equipped to diagnose and treat these patients. Herein, we aim to review clinical findings of brain imaging techniques in a specific subset of psychiatric disease states. Of particular interest is to examine the hypothetical implications of these discoveries in forensic psychiatry.

Received 03/07/2014 Review began 03/09/2014 Review ended 04/29/2014 Published 04/30/2014

C) Copyright 2014

Jacob. This is an open access article distributed under the terms of the Creative Commons Attribution License CC-BY 3.0., which permits unrestricted use, distribution, and reproduction in any medium, provided the original author and source are credited.
Categories: Neurology, Psychiatry, Radiology

Keywords: alzheimer's disease, brain disorders, neuro imaging, radiology, neurodegenerative disease, psychiatry, forensic psychiatry

\section{Introduction And Background}

\section{Etiology and pathophysiology of Alzheimer's disease}

The National Institute of Neurological and Communicative Disorders and Stroke has identified Alzheimer's Disease (AD) as a dual clinicopathological entity which must meet an everchanging set of criteria. Cummings, et al. report two essential elements, which must be met to securely identify AD: 1) identifiable neuropathological changes which include neurofibrillary tangles and senile plaques, causing neuronal atrophy and synaptic loss, and 2) clinical symptoms which demonstrate an impairment of cognitive abilities, such as improper functioning of working memory and episodic memory impairment [1]. An estimated 5.4 million Americans have $\mathrm{AD}$, with an additional 10 million coming patients from the baby boomer generation. Statistically, someone in America develops AD every 68 seconds. By 2050, there is expected to be one new case of $\mathrm{AD}$ every 33 seconds, or nearly a million new cases per year, and $\mathrm{AD}$ prevalence is projected to be 11 million to 16 million. The economic burden of $\mathrm{AD}$ on the 
healthcare system is palpable, with $\mathrm{AD}$ being the sixth leading cause of death in the United States. As we battle to decrease the mortality rates due to other disease states, AD presents an ever-increasing death rate and devastating level of morbidity. Between 2000 and 2008, the proportion of deaths due to heart disease, stroke, and prostate cancer decreased by $13 \%, 20 \%$, and $8 \%$, respectively. In comparison, the proportion of deaths due to AD increased by $66 \%$ [5].

The ability to obtain a neuropathological confirmation of $\mathrm{AD}$ is usually impossible during a patient's lifetime [2]. Thus, the clinical symptomatic expressions of the disease provide the strongest evidence for a clinician to diagnose AD [3]. However, the surge of biotechnologies, and specifically radiological sciences, within the neuroscientific community has allowed the biomedical community to develop a new set of ground rules for the clinical diagnoses of AD. In 2007, the International Working Group for New Research Criteria for the Diagnosis of AD proposed a new diagnostic framework [4]. The aim was to extend the limiting criteria put in place by the National Institute of Neurological and Communicative Disorders and StrokeAlzheimer's Disease and Related Disorders Association (NINCDS-ADRDA) [4]. The newly developed criterion for $\mathrm{AD}$ inculcates the worlds of symptomatic expression and biological evidence. The necessity for in vivo pathological evidence for $\mathrm{AD}$ permits a refined and more accurate method by which we may diagnose and subsequently treat $\mathrm{AD}$ patients. Yet, there still remains room for technology to advance our capabilities in diagnosing $\mathrm{AD}$ patients; for even with these newly refined guidelines, there is only a probable certainty that a patient has AD when diagnosed by a clinician. Conventionally, physicians use the term $\mathrm{AD}$ to characterize an abnormal amnestic disorder coupled with a variety of cognitive, neuropsychiatric, and behavioral aberrations.

The last two decades has enabled scientists and clinicians alike to utilize increasingly interventional procedures to obtain a more confident diagnosis of AD. Jagust, et al. report how reliable biomarkers used to validate a pathophysiology underlying $\mathrm{AD}$ is becoming increasingly possible [6]. The incorporation of reliable biomarkers to identify brain matter, which is expressive of $\mathrm{AD}$ on a micro-level scale, creates a paradigm shift in how we view and diagnose $\mathrm{AD}$, as it allows for the creation of a dual entity requirement for the precise diagnoses of $\mathrm{AD}$ : biologics and symptoms. Clark, et al. demonstrate the further incorporation of neuroimaging techniques and beta-amyloid biomarkers to validate the presumed diagnosis of AD [7]. Klunk, et al. have demonstrated the efficacy of using PiB PET amyloid imaging to concretely identify abnormalities in specific neuronal regions [8]. These reports support the idea that neuroimaging biomarkers are highly consistent with neuropathological lesions of AD.

The brain matter of $\mathrm{AD}$ patients presents two distinct qualities, which can be identified via biotechnological advances, imaging, and biomarker development. Understanding the difference between these two pathophysiologies allows for the refined development of markers, which will specifically target these specific abnormalities in a given brain: A) the amyloidosis path leading to neuritic plaques and B) the tauopathy path developing into neurofibrillary tangles [9]. Biomolecular techniques include CSF measures of a reduced amyloid beta concentration, increased total concentration of tau protein, and increased phospo-tau [10-13]. Predictive models have also proven that CSF biomarkers, which demonstrate an abnormal ratio between tau and amyloid beta, are reliably associated with extremely high rates of progression of mild cognitive impairment to $\mathrm{AD}$ [14-15]. According to studies by Blennow, et al., in vivo pathophysiological markers demonstrate consistent correlation with their partnered neuropathological lesions. These include PiB-PET and CSF amyloid beta for senile plaques [17] and total tau and phospho-tau concentration ratios with neurofibrillary tangles (Figure 1) [18]. A conjugation of a neurobiologically-based definition of AD coupled with clinical symptoms of cognitive impairment redefines and refines the entire field of $\mathrm{AD}$ research in that the availability of in vivo biomarkers now may form the basis of new research criteria. 


\section{Cureus}

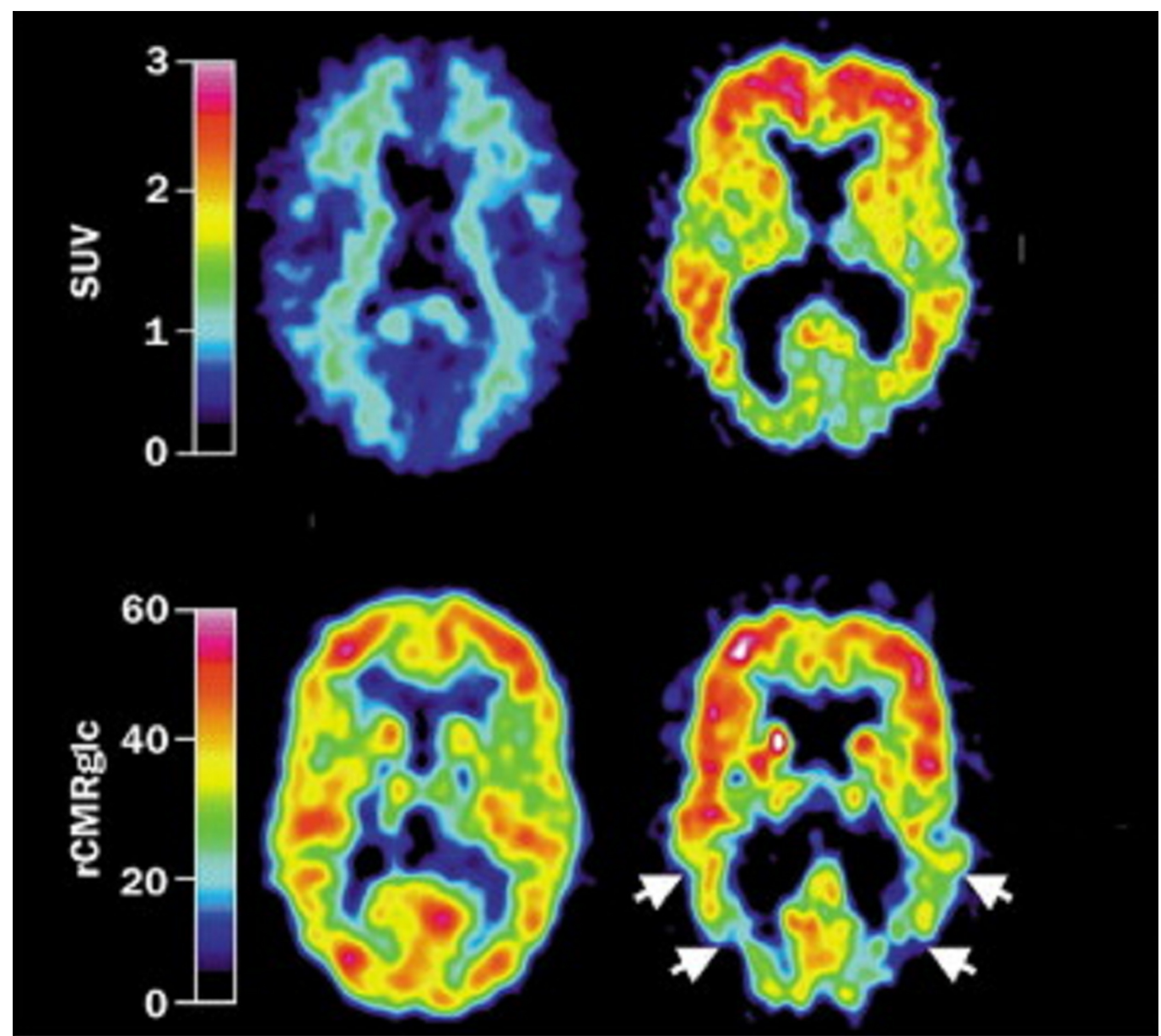

FIGURE 1: Functional Imaging Analysis of Neurodegenerative

\section{Brain}

C-PIB standardised uptake value (SUV) images. A clear difference is detected between the 11CPIB retention in the brain of a 67-year-old healthy person (left) and a 79-year-old AD patient (right). Bottom: 18F-FDG imaging analysis demonstrates altered retention of PIB in the frontal and temporoparietal regions of $A D$ patients. Used with permission from Elsevier, the Lancet Neurology [16]

\section{Review}

\section{Functional and anatomical imaging in Alzheimer's disease}

In 1991, Braak, et al. demonstrated initial abnormalities found within the actual brain structure of $\mathrm{AD}$ patients [19]. This work demonstrated the natural brain lesion development that occurs in $\mathrm{AD}$, beginning with neurofibrillary involvement of the entorhinal cortex and the hippocampus, as well as abnormal structural formation in the medial temporal lobes, which subsequently spreads to the neocortical association areas and the gradual cortical deposition of amyloid plaques [19]. In 1901, Alois Alzheimer first discussed the presence of amyloid in a causative form of dementia [20]. In this groundbreaking work, the German scientist assessed a 51-year-old woman who had presented with memory impairments, and allocated the cause of the symptoms to the buildup of amyloid plaques and neurofibrillary tangles in the brain. Over a century has gone by, and our therapeutic capabilities hinge on our diagnostic powers in detecting the presence of amyloid plaques earlier on in the progression of this disease (Figure 2). 


\section{Cureus}

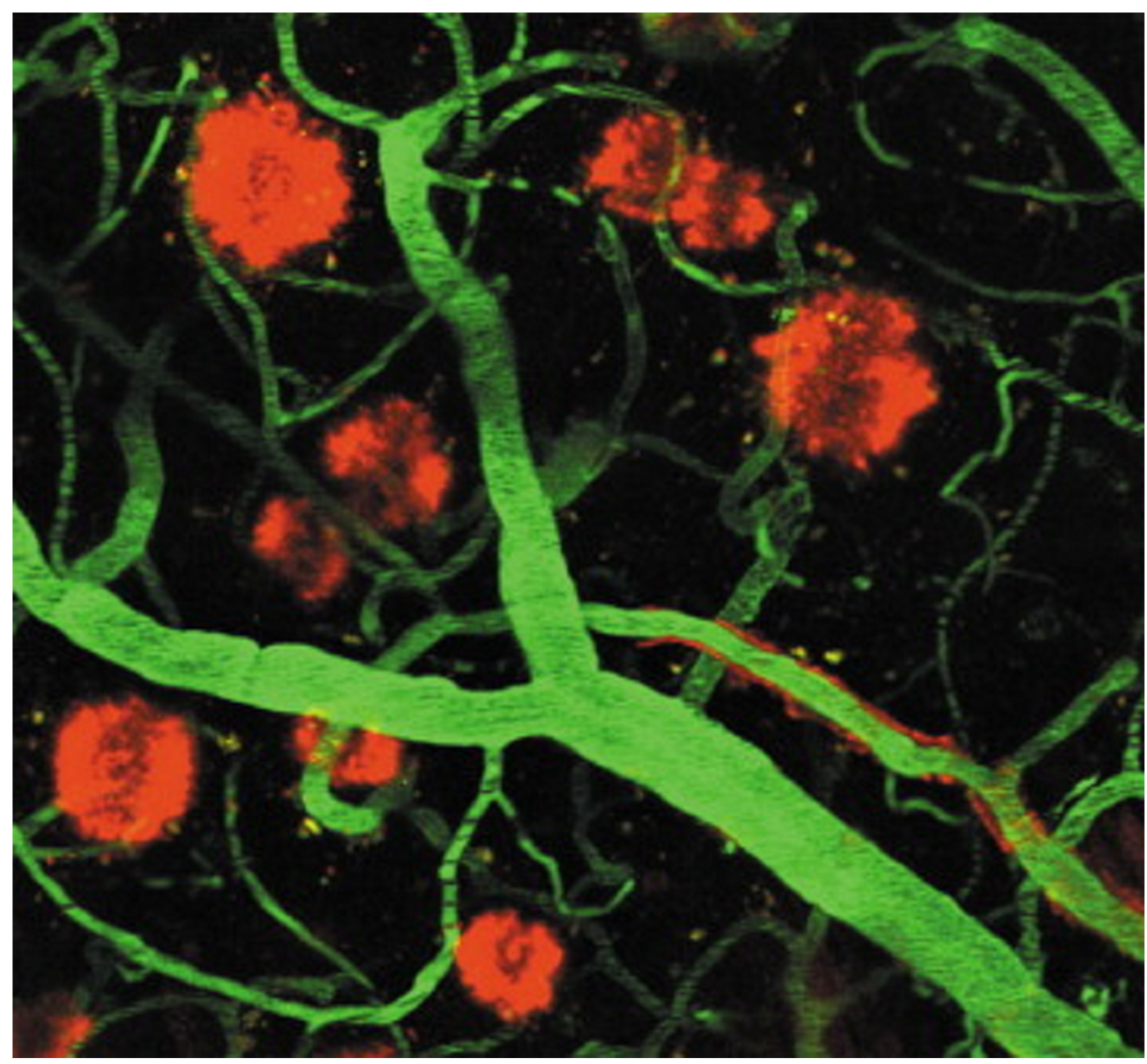

FIGURE 2: Cellular View of Modeled Neurodegenerative Mouse

\section{Brain}

Multiphoton microscopy of senile plaques in the brain of a live 21-month-old transgenic APP mouse. Courtesy of Dr Brian Bacskai, Department of Neurology, Massachusetts General Hospital, Charlestown, USA. Reused with licensed permission from Elsevier [16].

The advent of biomedical imaging processes and computerized algorithms powered the ability to image the brain in exquisite detail. Of utmost importance in the realm of biomedical imaging is the development of specific ligands, which can successfully deploy images of the presence of amyloid in particular regions of the brain. The last decade has seen the development of varying compounds that may be used for amyloid imaging in the brain. Nordberg reports that the genesis of plaque-binding compounds began with monoclonal antibodies against amyloid beta and peptide fragments [21], followed by radiolabelled analogues of thioflavin and chrysamine$\mathrm{G}$, which can be utilized in both PET and single-photon emission computed tomography (SPECT) imaging [22]. The clinical utilization of PET techniques in amyloid imaging has hitherto been unsuccessful. Friedland, et al. developed antibodies to amyloid beta labelled with technetium-99. They reported that that the monoclonal antibody $10 \mathrm{H} 3$ had the potential to be a successful amyloid imaging agent; however, when this compound was given to six AD patients, there was no cerebral uptake traced [23]. Further to this failure, SPECT imaging was used with iodine-123-labelled serum amyloid P component (SAP) in AD patients by Lotat, et al., and no binding of SAP was observed in any patients [24]. Despite these imaging limitations, Shoghi-Jadid, et al. aimed at in vivo imaging of amyloid beta in AD patients by utilizing F-FDDNP, a positron emission tomography (PET) tracer for determining amyloid 
plaques and neurofibrillary tangles in the brain in vivo. They administered this compound to nine $\mathrm{AD}$ patients, each suffering from varying degrees of cognitive impairment. Their results demonstrated that "the retention of F-FDDNP in the temporal, parietal, frontal, and occipital cortical regions were $10-15 \%$ higher than in the pons" [25]. In these studies, a 30\% higher retention rate of the compound was detected in the hippocampus, amygdala, and entorhinal cortex, areas that are known as neuronal circuits for a vast array of cognitive functions [25]. Klunk, et al. conducted one of the first human trials attempting to image amyloid plaques with C-PIB. After administering the compound to $16 \mathrm{AD}$ patients and nine subject controls, a significant difference in the retention rate of the compound was detected between both groups. The retention in patients was increased by a factor of nine when compared to controls. Regions which demonstrated this alteration were specific to the frontal cortex, parietal, occipital, and temporal cortices [26].

$\mathrm{Du}$, et al. applied magnetic resonance imaging methodologies to acquire precise volumetric changes of the entorhinal cortex (ERC) and hippocampus in $\mathrm{AD}$ patients. The study assessed 29 patients with $\mathrm{AD}$ and utilized manual measurements to obtain the volumes of the ERC and hippocampus based on coronal T1 weighted MR images. A significant 39\% decrease in the volume of the ERC was demonstrated with MR images, with a concomitant $27 \%$ decrease in the hippocampal region [27]. When compared with controls, AD patients also demonstrated decreased cortical grey matter when compared with controls. This research demonstrates the ability for MR imaging techniques to begin to develop concrete neuro-anatomical signs of $\mathrm{AD}$ [27] and establishes a positive relationship with recent studies by Braak, et al., which demonstrate a degenerative progress in $\mathrm{AD}$ beginning in the ERC and hippocampus [28]. Significantly, Du, et al. discovered within their studied AD cohort that variances of the ERC were more significant than hippocampal alterations, although both varied when compared to controls. Consistent findings of MR studies in AD patients illustrate atrophy of the ERC and hippocampus [29-32] as well to global brain atrophy, a decrease in grey matter, and a substantial increase in lesions of the white matter [33]. Several important studies, which aim to decipher the power of utilizing these specific discrepancies in the brains' of AD patients, need to be highlighted. Bobinski, et al. demonstrated that abnormalities of the ERC were readily and more easily detected in $\mathrm{AD}$ patients than abnormal hippocampal regions when compared to normal controls [34]. However, contrary to Bobinski's findings, Frisoni, et al. demonstrated that the hippocampal aberrations of AD patients is more prevalent than ERC changes. Frisoni, et al. reported increased diagnostic accuracy when using hippocampal volumetric abnormalities rather than ERC changes in AD patients [35].

The introduction of functional imaging techniques in the clinical and diagnostic setting has enabled the biomedical community to develop methods by which they may detect $\mathrm{AD}$ at an earlier stage of its development. FDG-PET imaging is utilized to measure cerebral metabolic rates of glucose within a physiological system. Given that the brain utilizes glucose at an increased ratio rate than the rest of the body, this imaging technique proves to be successful in creating refined images of $\mathrm{AD}$ in the brain. Tracking the glucose metabolic rates within neuronal circuits is a good measure for synaptic activity, neuronal health, and synaptic density [36]. To date, several animal and human studies have introduced this advanced methodology to study the molecular dynamics of $\mathrm{AD}$, as well as provide a basis for concise $\mathrm{AD}$ diagnostics.

Both qualitative and quantitative alterations in the AD brain have been discovered with FDGPET. A common denominator in clinical studies of FDG-PET in AD is the presence of global metabolic deficits throughout the neocortex [37]. An AD metabolic pattern has been outlined by Mosconi, et al. to include hypometabolism in associative parietotemporal areas, the posterior cingulate cortex, and the precuneus [38]. As the disease progresses, hypometabolic irregularities are traced by FDG-PET to spread to the prefronal cortex [38]. In agreement with studies performed by MRI techniques, FDG-PET consistently demonstrates metabolic irregularities in the entorhinal cortex and the hippocampus [39]. Frontotemporal lobar 
degeneration is also a common neuroanatomical manifestation of dementia-related disorders, including AD. FDG-PET has demonstrated that in AD patients there exists metabolic impairment in the frontal and anterior temporal lobes [40-41]. Milder hypometabolic rates are detected in the parietal lobes at the early stages of $\mathrm{AD}$, which progressively become more evident as the disease manifests on a symptomatic scale over time.

The clinical treatment of $\mathrm{AD}$ does not end with a diagnosis from a radiological perspective. The advantage of FDG-PET imaging in comparison to other modalities hoping to diagnose and treat $\mathrm{AD}$ patients is that FDG-PET contains the highest specificity in tracking the progression of the disease throughout other regions of the brain. This allows for the monitoring of the efficacy of treatments, as well as an avenue into personalized medicine for the treatment of AD.

\section{Conclusions}

The roles of impairment in the ERC is crucial in understanding behavior, particularly impairments in readiness, emotional dysregulation, and risk awareness. Each of these symptoms have significant forensic implications. So, too, the role of the temporal lobe impairment in creating obsessive compulsive symptoms, at times of a sexual nature, and problems with autobiographical memory is only beginning to be understood.

Neuropsychological testing, the current gold standard in quantifying cognitive symptoms and resultant behavior, has not yet begun to effectively tackle most areas of the brain, certainly not areas of the ERC and medial temporal lobes. The combination of thorough social history, neuropsychological testing, and this current surge forward in PET biotechnology may allow the courts to more confidently utilize neuroimaging to examine those parts of the brain most susceptible to creating abnormal behavior, areas which are currently beyond the purview of a thorough neuropsychological examination.

The move toward ecological validity, how does the testing translate in real world behavior, is most important in the tension among various neuroimaging techniques. How can this information be of service in helping the trier of fact parse out the complex factors found in many forensic cases?

Relying upon clinically proven techniques is important. As we understand the role of brain structures in diseases like $\mathrm{AD}$, and correlate the cognitive impairments and behavioral abnormalities created by the deterioration of these brain structures, the ability to generalize those findings to others with similar impairment, but not $\mathrm{AD}$, will provide support that many of these cognitive deficits are final common pathways to aberrant behavior.

\section{Additional Information}

\section{Disclosures}

Conflicts of interest: In compliance with the ICMJE uniform disclosure form, all authors declare the following: Payment/services info: All authors have declared that no financial support was received from any organization for the submitted work. Financial relationships: All authors have declared that they have no financial relationships at present or within the previous three years with any organizations that might have an interest in the submitted work. Other relationships: All authors have declared that there are no other relationships or activities that could appear to have influenced the submitted work.

\section{Acknowledgements}

I would like to thank George Woods, MD, for providing valuable insight and guidance in the development of this paper. 


\section{References}

1. Cummings JL: Alzheimer's disease. N Engl J Med. 2004, 351:56-67.

2. Querfurth HW, LaFerla FM: Alzheimer's disease. N Engl J Med. 2010, 362:329-44. 10.1056/NEJMra0909142

3. McKhann G, Drachman D, Folstein M, Katzman R, Price D, Stadlan EM: Clinical diagnosis of Alzheimer's disease: report of the NINCDS-ADRDA Work Group under the auspices of Department of Health and Human Services Task Force on Alzheimer's Disease. Neurology. 1984, 34:939-44.

4. Dubois B, Feldman HH, Jacova C, et al.: Research criteria for the diagnosis of Alzheimer's disease: revising the NINCDS-ADRDA criteria. Lancet Neurol. 2007, 6:734-46.

5. Alzheimer's Association, Thies W, Bleiler L: 2011 Alzheimer's disease facts and figures . Alzheimers Dement. 2011, 7:208-44. 10.1016/j.jalz.2011.02.004

6. Jagust WJ, Landau SM, Shaw LM, et al.: Relationships between biomarkers in aging and dementia. Neurology. 2009, 73:1193-99.

7. Clark CM, Xie S, Chittams J, et al.: Cerebrospinal fluid tau and beta-amyloid: How well do these biomarkers reflect autopsy-confirmed dementia diagnoses?. Arch Neurol. 2003, 60:1696702 .

8. Ikonomovic MD, Klunk WE, Abrahamson EE, et al.: Post-mortem correlates of in vivo PiB-PET amyloid imaging in a typical case of Alzheimer's disease. Brain. 2008, 131:1630-45.

10.1093/brain/awn016

9. National Institute on Aging, Reagan Institute Working Group on Diagnostic Criteria for the Neuropathological Assessment of Alzheimer's Disease: Consensus recommendations for the postmortem diagnosis of Alzheimer's disease. The National Institute on Aging, and Reagan Institute Working Group on Diagnostic Criteria for the Neuropathological Assessment of Alzheimer's Disease. Neurobiol Aging. 1997, 18:S1-2.

10. Strozyk D, Blennow K, White LR, Launer LJ: CSF A 342 levels correlate with amyloidneuropathology in a population-based autopsy study. Neurology. 2003, 60:652-6.

11. Blennow K, Hampel H, Weiner M, Zetterberg H: Cerebrospinal fluid and plasma biomarkers in Alzheimer disease. Nat Rev Neurol. 2010, 6:131-44. 10.1038/nrneurol.2010.4

12. Hampel H, Burger K, Pruessner JC, et al.: Correlation of cerebrospinal fluid levels of tau protein phosphorylated at threonine 231 with rates of hippocampal atrophy in Alzheimer disease. Arch Neurol. 2005, 62:770-3.

13. Mattsson N, Zetterberg H, Hansson O, et al.: CSF biomarkers and incipient Alzheimer disease in patients with mild cognitive impairment. JAMA. 2009, 302:385-93. 10.1001/jama.2009.1064

14. Henneman WJ, Vrenken H, Barnes J, et al.: Baseline CSF p-tau levels independently predict progression of hippocampal atrophy in Alzheimer disease. Neurology. 2009, 73:935-40. 10.1212/WNL.0b013e3181b879ac

15. Ewers M, Buerger K, Teipel SJ, et al.: Multicenter assessment of CSF-phosphorylated tau for the prediction of conversion of MCI. Neurology. 2007, 69:2205-12.

16. Nordberg A: PET imaging of amyloid in Alzheimer's disease . Lancet Neurol. 2004, 3:519-27.

17. Tapiola T, Alafuzoff I, Herukka SK, et al.: Cerebrospinal fluid $\beta$-amyloid 42 and tau proteins as biomarkers of Alzheimer-type pathologic changes in the brain. Arch Neurol. 2009, 66:382-89.

18. Buerger K, Ewers M, Pirttila T, et al. : CSF phosphorylated tau protein correlates with neocortical neurofibrillary pathology in Alzheimer's disease. Brain. 2006, 129:3035-41.

19. Braak H, Braak E: Neuropathological stageing of Alzheimer-related changes. Acta Neuropathol. 1991, 82:239-59.

20. Alzheimer A: Über einen eigenartigen schweren Erkrankungsprozess der Hirnrinde . Neurologisches Centralblatt. 1906, 23:1129-36.

21. Lee HJ, Zhang Y, Zhu C, Duff K, Pardridge WM: Imaging brain amyloid of Alzheimer disease in vivo in transgenic mice with an Abeta peptide radiopharmaceutical. J Cereb Blood Flow Metab. 2002, 22:223-31.

22. Zhuang ZP, Kung MP, Wilson A, et al.: Structure-activity relationship of imidazo[1,2a]pyridines as ligands for detecting beta-amyloid plaques in the brain. J Med Chem. 2003, 46:237-43.

23. Friedland RP, Kalaria R, Berridge M, et al.: Neuroimaging of vessel amyloid in Alzheimer's disease. Ann N Y Acad Sci. 1997, 826:242-7.

24. Lovat LB, Persey MR, Madhoo S, Pepys MB, Hawkins PN: The liver in systemic amyloidosis: 
Insights from 123I serum amyloid P component scintigraphy in 484 patients. Gut. 1998, 42:727-34.

25. Shoghi-Jadid K, Small GW, Agdeppa ED, et al.: Localization of neurofibrillary tangles and beta-amyloid plaques in the brains of living patients with Alzheimer disease. Am J Geriatr Psychiatry. 2002, 10:24-35.

26. Klunk WE, Engler H, Nordberg A, et al.: Imaging brain amyloid in Alzheimer's disease with Pittsburgh Compound-B. Ann Neurol. 2004, 55:306-19.

27. Du AT, Schuff N, Amend D, et al.: Magnetic resonance imaging of the entorhinal cortex and hippocampus in mild cognitive impairment and Alzheimer's disease. J Neurol Neurosurg Psychiatry. 2001, 71:441-7.

28. Braak H, Braak E, Bohl J: Staging of Alzheimer-related cortical destruction. Eur Neurol. 1993, 33:403-8.

29. Juottonen K, Laakso MP, Partanen K, et al.: Comparative MR analysis of the entorhinal cortex and hippocampus in diagnosing Alzheimer disease. AJNR Am J Neuroradiol. 1999, 20:139-44.

30. Jack CR Jr, Petersen RC, O'Brien PC, et al.: MR-based hippocampal volumetry in the diagnosis of Alzheimer's disease. Neurology. 1992, 42:183-8.

31. Schuv N, Amend D, Ezekiel F, et al.: Change of hippocampal N-acetyl aspartate and volume in Alzheimer's disease. Neurology. 1997, 49:1513-21.

32. Tanabe JL, Amend D, SchuV N, et al.: Tissue segmentation of the brain in Alzheimer's disease . AJNR Am J Neuroradiol. 1997, 18:115-23.

33. Barber R, Scheltens P, Gholkar A, et al.: White matter lesions on magnetic resonance imaging in dementia with Lewy bodies, Alzheimer's disease, vascular dementia, and normal aging. J

Neurol Neurosurg Psychiatry. 1999, 67:66-72.

34. Bobinski M, de Leon MJ, Convit A, et al.: MRI of entorhinal cortex in mild Alzheimer's disease . Lancet. 1999, 353:38-40.

35. Frisoni GB, Laakso MP, Beltramello A, et al.: Hippocampal and entorhinal cortex atrophy in frontotemporal dementia and Alzheimer's disease. Neurology. 1999, 52:91-100.

36. Attwell, D, Iadecola C: The neural basis of functional brain imaging signals . Trends Neurosci. 2002, 25:621-625.

37. Silverman DHS, Small GW, Phelps ME: Clinical value of neuroimaging in the diagnosis of dementia. Sensitivity and specificity of regional cerebral metabolic and other parameters for early identification of Alzheimer's disease. Clin Positron Imaging. 1999, 2:119-130.

38. Mosconi L: Brain glucose metabolism in the early and specific diagnosis of Alzheimer's disease. FDG-PETstudies in MCI and AD. Eur J Nucl Med Mol Imaging. 2005, 32:486-510.

39. Desanti S, DeLeon L, Rusinek H, et al.: Hippocampal formation glucose metabolism and volume losses in MCI and AD. Neurobiol Aging. 2001, 22:529-39.

40. Diehl-Schmid J, Grimmer T, Drzezga T, et al.: Decline of cerebral glucose metabolism in frontotemporal dementia: a longitudinal 18F-FDG-PET-study. Neurobiol Aging. 2007, 28:

41. Jeong Y, Cho SS, Park JM , et al.: 18F-FDG PET findings in frontotemporal dementia: an SPM analysis of 29 patients. J Nucl Med. 2005, 46:233-9. 